Proc. of the 13th Symposium of Magnetic Measurements and Modeling SMMM'2018, Wieliczka, Poland, October 8-10, 2018

\title{
Magnetic Relaxation in Iron Based Melt Spun Ribbons
}

\author{
P. KWAPUlińsKi* AND G. HANECZOK \\ University of Silesia, Institute of Materials Science, 75 Pułku Piechoty 1A, 40-500 Chorzów, Poland \\ In this paper magnetic relaxation processes in iron based amorphous alloys are examined in order to determine \\ thermal/time instability of magnetic properties at room temperature. It was shown that the reversible component \\ of magnetic relaxation in $\mathrm{Fe}_{74} \mathrm{Cu}_{1} \mathrm{Cr}_{3} \mathrm{Si}_{13} \mathrm{~B}_{9}$ alloy can be well described by the coupling model usable for diffusion \\ in strongly correlated systems. The proposed approach allows monitoring initial stages of structural relaxation.
}

DOI: 10.12693/APhysPolA.136.701

PACS/topics: 61.43.Dq, 66.30.-h, 75.50.Kj, 75.60.Lr

\section{Introduction}

It is well known that iron-based amorphous alloys obtained by melt spinning technique belong to a modern family of very soft magnetic materials. Low field magnetic permeability of these materials can be of the order of $10^{4}$ and coercivity below $1 \mathrm{~A} / \mathrm{m}[1-5]$. Such a softening is due to formation of a non-equilibrium amorphous phase free of magnetic anisotropy. The most important disadvantage of amorphous alloys are the temperature and/or time instabilities of macroscopic properties observed even at room temperatures. Indeed, due to fast cooling from liquid phase amorphous material is not in thermodynamic equilibrium and in general tends to equilibrium via two processes - structural relaxation and crystallization [1-5]. The structural relaxation is mainly caused by diffusion of free volume frozen into material during fabrication. In order to describe such diffusion the concept of the Kronmüller pairs defined in [6] (see Fig. 4 in [6]) is usually used in literature. In the vicinity of free volume a group of atoms form more or less stable configuration and at given temperature one atom can change its position to a neighboring one via a correlated transition through a free volume. These transitions being the source of the structural relaxation cause a local change of material density, internal stresses, magnetic coupling, etc., and in consequence lead to some changes of macroscopic material properties like magnetic permeability. It is generally accepted that the structural relaxation consists of two components - reversible one and irreversible one. Both of these components can be suppressed by applying annealing at elevated temperatures. Thus, application of any amorphous alloys requires a detailed examination of the thermal/time instabilities and their influence on the operational parameters. The goal of the present paper is to study magnetic relaxation for iron based amorphous alloy $\mathrm{Fe}_{74} \mathrm{Cu}_{1} \mathrm{Cr}_{3} \mathrm{Si}_{13} \mathrm{~B}_{9}$ and test the applicability of the coupling model suitable for diffusion in correlated systems.

*corresponding author; e-mail: piotr.kwapulinski@us.edu.pl

\section{Theoretical background}

In strongly correlated systems one can define the so-called anomalous diffusion exponent $d$ according to the relation [7]:

$$
\left(\sqrt{\left\langle x^{2}\right\rangle}\right)^{d} \propto t
$$

where $x$ and $t$ denotes diffusion path and time, respectively. The case $d=2$ corresponds to a random walk for which we have $[7,8]$ :

$$
\frac{\left\langle x^{2}\right\rangle}{t} \propto D \propto W
$$

where $D$ is the diffusion coefficient and $W$ is the transition rate of the diffusing object from one stable position to the neighboring one. It is worth mentioning that Eq. (2) can be used as a definition of $D$ valid for any system. According to Eqs. (1) and (2) in correlated system the transition rate is time dependent, i.e., [8-11]:

$$
W \propto t^{\left(\frac{2}{d}-1\right)} \text { or } W=W_{0}\left(\frac{t}{t_{C}}\right)^{-n},
$$

where $W_{0}$ is unaffected transition rate, $t_{C}$ is the scaling time and the exponent $n=1-2 / d$ is called the coupling parameter. Let us notice that $n$ varies between 0 (for $d=2$ (random walk)) and 1 (for $d \rightarrow \infty$ (totally correlated system)).

Kinetic equations describing a variation of $n_{\mu}$ population of diffusing objects in $\mu$ positions disturbed by application of a weak external field ( $\mu \rightarrow \nu$ transitions) in the frame of two-level model are

$$
\frac{\mathrm{d} n_{\mu}}{\mathrm{d} t}=W_{\mu, \nu} n_{\nu}-W_{\nu, \mu} n_{\mu}
$$

and can be solved assuming that $n_{\mu}+n_{\nu}=n_{0}\left(n_{0}\right.$ is the number of diffusing objects) and $W_{\mu, \nu}=W_{\nu, \mu}=$ $W_{0}\left(\frac{t}{t_{c}}\right)^{-n}$ with the initial condition that at time $t=0$ the population is equally distributed between sites, i.e., $n_{\mu}=n_{\nu}=n_{0} / 2$. The corresponding solutions can be written in the form

$$
n_{\mu}=\frac{n_{0}}{2}-\frac{n_{0}}{2} \beta h \exp \left(-\left(\frac{t}{\tau}\right)^{1-n}\right)
$$


and

$$
n_{\nu}=\frac{n_{0}}{2}+\frac{n_{0}}{2} \beta h \exp \left(-\left(\frac{t}{\tau}\right)^{1-n}\right),
$$

where $\beta=1 / k_{\mathrm{B}} T$ ( $k_{\mathrm{B}}$ is the Boltzmann constant, $T$ is the temperature) and $h$ is the difference in activation enthalpies of sites $\mu$ and $\nu$ caused by the perturbation field. The relaxation time $\tau$ is given by

$$
\tau=\left(\frac{(1-n)}{2 W_{0}} t_{c}^{-n}\right)^{\frac{1}{1-n}} .
$$

Taking into account the above, one can write the formula describing magnetic relaxation in strongly correlated systems, i.e. the reluctivity $r(t)$ takes the form [12-15]:

$$
r(t)=r_{0}+I_{C}\left(1-\exp \left(-(t / \tau)^{1-n}\right)\right),
$$

where $r_{0}$ is $r(0)$ and $I_{C}$ is the relaxation intensity. Let us notice that for $n=0$ (uncorrelated system) the transition rate is time independent $W=W_{0}$, the relaxation time $\tau=1 / 2 W_{0}$ and $r(t)$ simplifies to the wellknown Debye relaxation.

Based on the presented formalism for magnetic relaxation in amorphous alloys we propose the following formula [12-15]:

$$
\begin{aligned}
& r(t)=r_{0}+I_{C}\left[1-\exp \left(-\left(t / \tau_{C}\right)^{1-n}\right)\right] \\
& \quad+I_{I}\left[1-\exp \left(-t / \tau_{I}\right)\right],
\end{aligned}
$$

where the first component describes the reversible component observed just after demagnetization (coupling model with the intensity $I_{C}$ and the relaxation time $\tau_{C}$ ) and the second component describes the irreversible (long-time) component (the Debye relaxation with the intensity $I_{I}$ and the relaxation time $\tau_{I}$ ).

\section{Experimental}

Experiments were carried out for $\mathrm{Fe}_{74} \mathrm{Cu}_{1} \mathrm{Cr}_{3} \mathrm{Si}_{13} \mathrm{~B}_{9}$ amorphous melt spun ribbons with thickness and width of about $20 \mu \mathrm{m}$ and $5 \mu \mathrm{m}$, respectively. In order to study the structural relaxation in the context of free volume diffusion samples in the as-quenched state were annealed at temperatures ranging from $300 \mathrm{~K}$ to $650 \mathrm{~K}$ for one hour. Such annealing slightly changes the amorphous microstructure and, what follows, changes the degree of the advancement of structural relaxation. Measurements of magnetic reluctivity at low field $(0.1 \mathrm{~A} / \mathrm{m})$ versus time at room temperature were carried out for samples after demagnetization by applying precision RLC meterAgilent E4980A. Special attention was paid to stabilize the temperature of measurements $300 \mathrm{~K}$.

\section{Results and discussion}

Figure 1 shows three curves of magnetic reluctivity vs. time measured after demagnetization for samples preliminary annealed at temperatures 323,423 , and $473 \mathrm{~K}$. One can see that the reluctivity varies with time reaching at least a partial saturation over $80 \mathrm{ks}(22 \mathrm{~h})$.

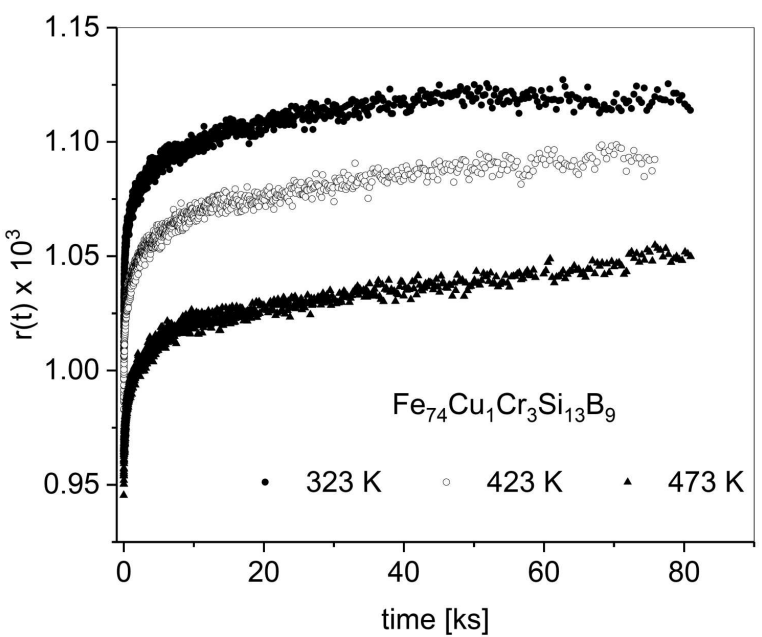

Fig. 1. Family of reluctivity vs. time curves measured at room temperature for samples of $\mathrm{Fe}_{74} \mathrm{Cu}_{1} \mathrm{Cr}_{3} \mathrm{Si}_{13} \mathrm{~B}_{9}$ amorphous melt spun ribbons after demagnetization.

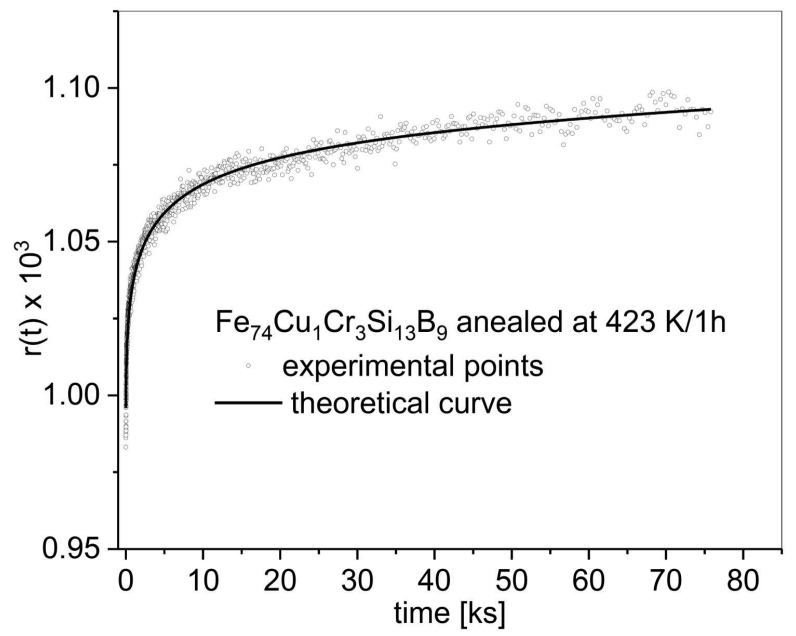

Fig. 2. Reluctivity vs. time $r(t)$ determined for $\mathrm{Fe}_{74} \mathrm{Cu}_{1} \mathrm{Cr}_{3} \mathrm{Si}_{13} \mathrm{~B}_{9}$ amorphous melt spun ribbons after demagnetization, preliminary annealed at $423 \mathrm{~K}$ for $1 \mathrm{~h}$ and theoretical curve calculated by applying Eq. (8).

The measured curves were numerically analyzed with the assumption that $r(t)$ is described by Eq. (8). In the analysis the standard fitting procedure was used and free parameters to be fitted are the following: $r(0)$, $I_{C}$ (intensity of the coupling process), $\tau_{C}$ (relaxation time of the coupling process), $n$ (coupling parameter describing a correlation degree), $I_{I}$ (intensity of the long-time irreversible process described by the Debye relaxation), and $\tau_{I}$ (relaxation time of the Debye relaxation). An example of the obtained fit goodness is presented in Fig. 2, where the curve $r(t)$ measured for sample preliminary annealed at $423 \mathrm{~K}$ for $1 \mathrm{~h}$ and the theoretical curve calculated by applying Eq. (8) are shown. Similar results have been obtained for the other measured curves. 


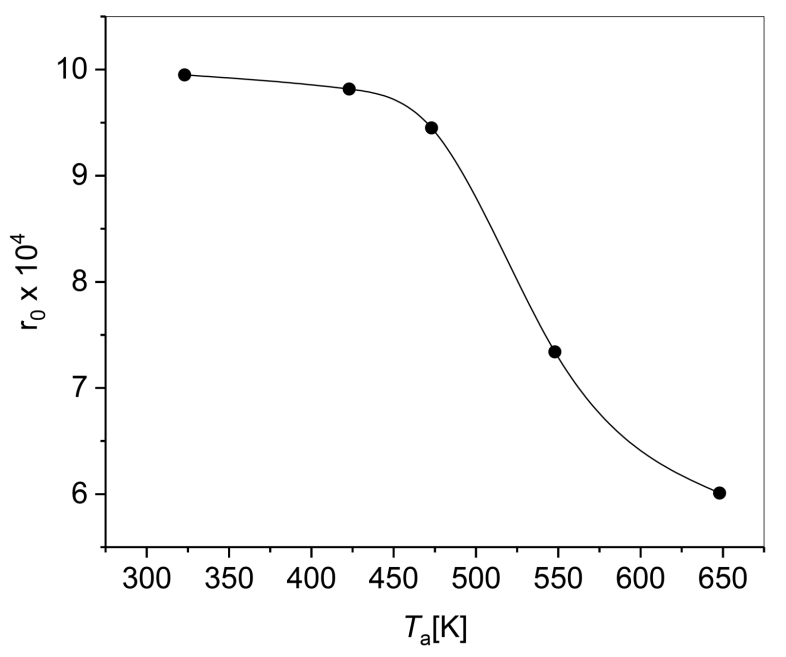

Fig. 3. Parameter $r_{0}$ (see Eq. (8)) plotted vs. annealing temperature $T_{a}$.

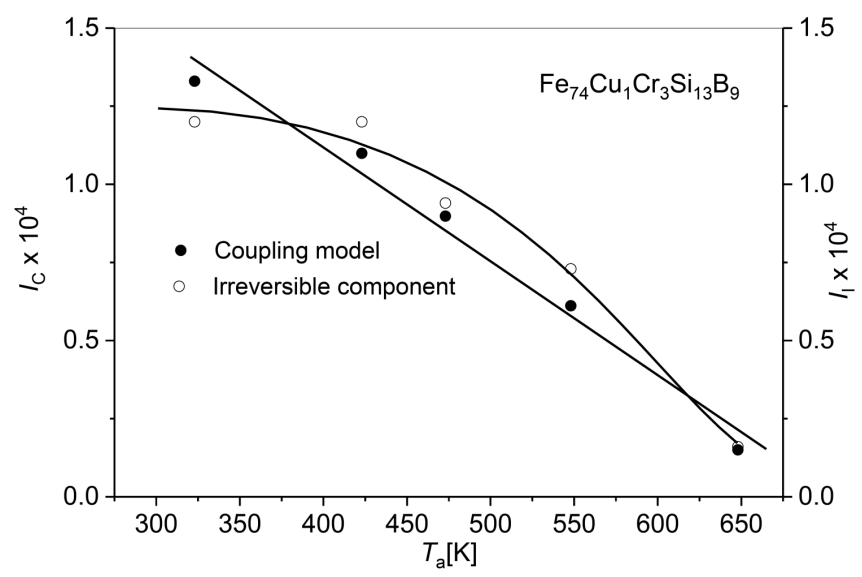

Fig. 4. Intensity of the coupling model (reversible component) and intensity of the irreversible component of magnetic relaxation plotted vs. annealing temperature $T_{a}$.

The results of numerical analysis are presented in Figs. 3-6, where the parameters of Eq. (8), i.e., $r_{0}$, intensities $I_{C}$ and $I_{I}$, relaxation times $\tau_{C}$ and $\tau_{I}$, and the coupling parameter $n$, are plotted vs. annealing temperature $T_{a}$. First of all we have to notice that the initial reluctivity $r_{0}$ decreases with increasing $T_{a}$ which corresponds to an increase of initial magnetic permeability. This effect is known in literature and can be assigned to redistribution of free volume and related to this a decrease of internal stresses [1-6]. The data presented in Fig. 4 shows that both intensities of relaxation components decreases with increasing annealing temperature which obviously means that the applied preliminary annealing makes the examined material more stable. Let us notice that an additional increase of the preliminary annealing temperature is not an option because it may cause formation of a nanocrystalline phase. Figure 5 shows the relaxation times for both processes plotted vs. $T_{a}$. It is evident that $\tau_{C}$ strongly decreases with increasing $T_{a}$ which confirms that due to preliminary annealing the reversible component of magnetic relaxation disappears. The relaxation time of the irreversible component is about three orders of magnitude longer and according to Fig. 5 does not depend on $T_{a}$. This means that this component at short times (let say up to $10 \mathrm{ks}$ ) does not play any significant role and at long times (over $40 \mathrm{ks}$ ) can be approximated by a straight line [15].

Figure 6 shows the coupling parameter $n$ plotted vs. $T_{a}$. The observed decrease of $n$ from 0.7 for the as-quenched state to about 0.5 for the sample annealed at $650 \mathrm{~K} / \mathrm{h}$ indicates that the amorphous structure due to free volume diffusion (leading to their redistribution) releases internal stresses and causes an increase of magnetic coupling. The latter is confirmed by a decrease

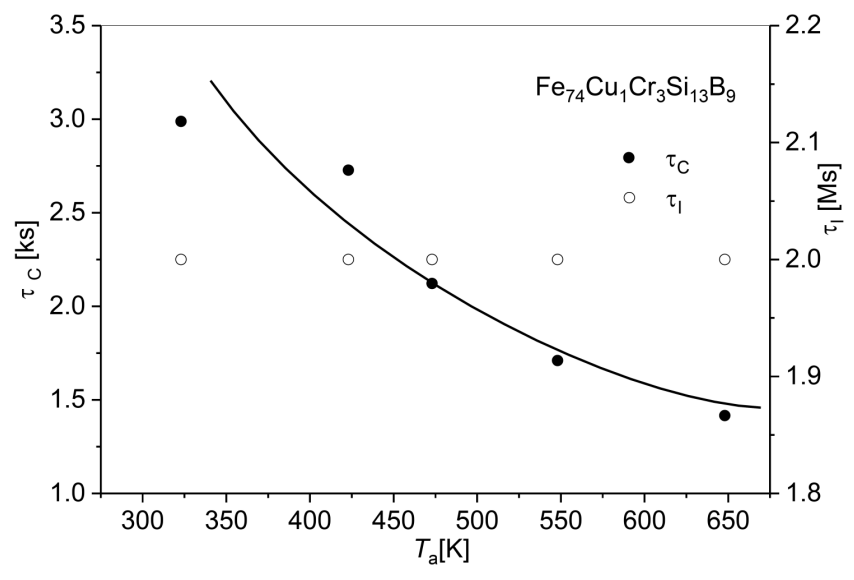

Fig. 5. Relaxation time of the coupling model (reversible component) and relaxation time of the irreversible component of magnetic relaxation plotted vs. annealing temperature $T_{a}$.

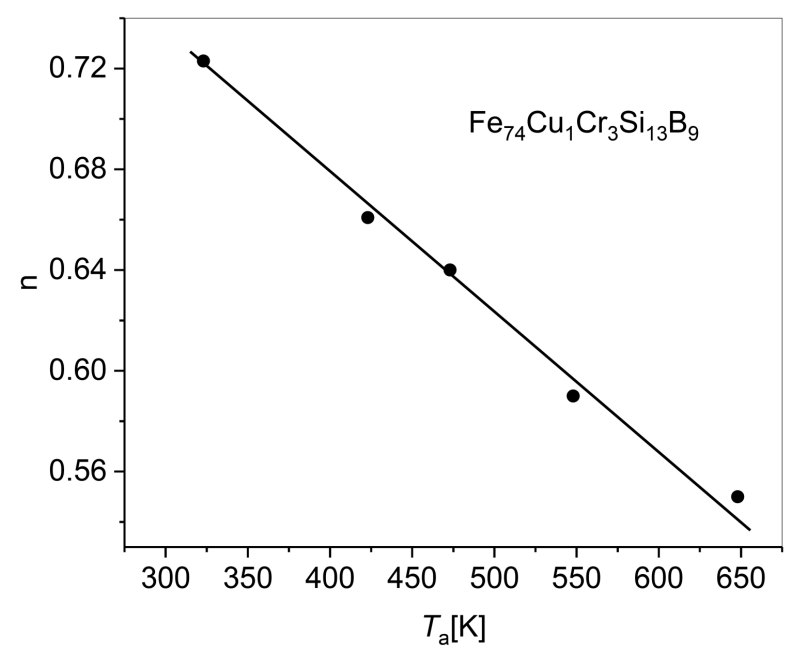

Fig. 6. Coupling parameter $n$ plotted vs. annealing temperature $T_{a}$. 
of magnetic reluctivity (or an increase of magnetic permeability) shown in Fig. 3. Such a behavior is known as formation of the so-called relaxed amorphous phase (step by step) [1-5]. It has to be stressed that the measurements of magnetic relaxation and the presented numerical analysis allows monitoring the first stages of structural relaxation and what follows is the longterm stability of macroscopic properties of iron base amorphous alloys.

\section{Conclusions}

The main conclusions of the present paper can be summarized as follows: (i) reversible component of magnetic relaxation in iron based amorphous alloys (e.g. $\mathrm{Fe}_{74} \mathrm{Cu}_{1} \mathrm{Cr}_{3} \mathrm{Si}_{13} \mathrm{~B}_{9}$ ) can be well described by the coupling model which allows monitoring initial stages of structural relaxation, (ii) relaxation time of the irreversible component of magnetic relaxation is at least three orders of magnitude longer than the relaxation time of the reversible component which means that for long times this component can be approximated by a straight line, (iii) the observed disappearance of thermal/time instabilities of magnetic properties caused by the preliminary annealing is quantitatively documented in Figs. 3-6.

\section{References}

[1] M.E. McHenry, M.A. Willard, D.E. Laughin, Prog. Mater. Sci. 44, 291 (1999).

[2] G. Haneczok, J. Rasek, Def. Diff. Forum 224, 13 (2004).
[3] Z. Stokłosa, J. Rasek, P. Kwapuliński, G. Badura, G. Haneczok, L. Pająk, J. Lelątko, A. Kolano-Burian, J. Alloys Comp. 509, 9050 (2011).

[4] Z. Stokłosa, J. Rasek, P. Kwapuliński, G. Haneczok, G. Badura, J. Lelątko, Mater. Sci. Eng. C 23, 49 (2003).

[5] A. Chrobak, G. Haneczok, Z. Stokłosa, P. Kwapuliński, J. Rasek, G. Chełkowska, Phys. Status Solidi A 196, 248 (2003).

[6] H. Kronmüller, Philos. Mag. B 48, 127 (1983).

[7] D. Stauffer, H.E. Stanley, From Newton to Mandelbrot, Springer, Berlin 1996.

[8] K.L. Ngay, in: Disorder Effects on Relaxation Processes, Eds. R. Richter, A. Blumen, Springer, Heidelberg 1994, p. 89.

[9] K.L. Ngay, A.K. Jonsher, G.T. White, Nature $\mathbf{2 7 7}$ 185 (1979).

[10] K.L. Ngay, G.T. White, Phys. Rev. B 20, 2475 (1979).

[11] J. Colmednero, A. Arbe, A. Algeria, Phys. Rev. Lett. 71, 2603 (1993).

[12] L. Nèel, J. Phys. Radium 13, 249 (1952).

[13] L. Nèel, J. Appl. Phys. Suppl. 30, S3 (1959).

[14] J. Rivas, M.A. Lopez-Quintela, D. Martinez, F. Waltz, H. Kronmüller, J. Non-Cryst. Solids 131, 1235 (1991)

[15] A. Chrobak, G. Chełkowska, G. Haneczok, P. Kubik, Ł. Madej, Acta Phys. Pol. A 115, 396 (2009). 